\title{
Various Recommender Systems and Their usage in the Domains of Mobile Based and Online Applications
}

\author{
Ushike Sudhakar Reddy, K.Uma Pavan Kumar, B.Priyanka
}

\begin{abstract}
The activities of the users are surrounded by online shopping, online content fetching and online payment of the various bills. The important point is in case of the online shopping with Amazon, Flipkart and many other online shopping sites provides some sort of intelligent assistance to the user. Based on the past history, based on the user profile. Such kind of the applications were named as recommender systems.

The most common categories of recommender systems involves, the collaborative filtering, content-based filtering, multi-criteria recommender systems, risk-aware recommender systems, mobile recommender systems and Hybrid recommender systems. The current work dealing with the process followed by the recommender systems along with various key factors embedded in the usage. The suggestions given by the application to the user depends on user profile, and content searched by the user and the collaboration of other products with the current product.

The work focus on the implementation algorithms existing in the process of recommender systems, the above listed categories of recommender systems follow certain key mechanisms depending on the user query. The work also deals with the performance aspects of the recommender systems in case of accuracy and reproducibility in recommender system research. Especially the mobile recommender systems there are certain limitations of region and accuracy of the results. Overall the outcome of the work is to describe the importance of the recommender systems and the internal mechanism followed by various recommender systems.
\end{abstract}

Keywords: Recommender System, User Profiling, Collaborative Filtering, Mobile recommender system, Intelligent assistance.

\section{INTRODUCTION}

Mobile based recommender systems gaining popularity as the usage of the mobiles become a generic in every day life. A recommender system basically emits the suggestions to the user based on the user profile, item based collaborative filtering and seed data.

The example real world recommender systems are amazon, Netflix and TripAdvisor. In case of amazon based on the user query related to a product many recommendations were presented.

For example the post: Machine Learning with Python outputs many books in the context of the machine learning, at

Revised Version Manuscript Received on September 10, 2019.

Ushike Sudhakar Reddy, Assistant Professor, CSE, Malla Reddy Institute of Technology, Hyderabad, Telangana, India..(Email: sudha.reddy500@gmail.com)

Dr.K.UmaPavanKumar, Associate Professor, CSE, Malla Reddy Institute of Technology, Hyderabad, Telangana, India..(Email: Dr.kethavarapu@gmail.com)

B.Priyanka, Assistant Professor, CSE, Malla Reddy Institute of Technology, Hyderabad, Telangana, India.(Email: Priyanka.biradar511@gmail.com) the moment if we select any of the book then the system displays the possible delivery date of the book based on the pin code and provides a description about the product(book in this case).

One of the interesting aspect is the system also suggests the selected product along with frequently bought together which gives a reference and similarity based suggestion to the user.

The other side of this recommendation is the system displays the other similar products that the customers also bought along with the selected product. The interesting fact is the system provides reviews about the product based on some parameters and the user can have the glance of un biased reviews and can conclude the buying of product, getting knowledge about frequently bought items with an additional information of reviews also.

Similar to the amazon, Netflix allows the users to get the recommendations based on the user profile like the kind of the movies, tv shows. It gives the rating information based on the reviews provided by the users and depending on the reviews the user can watch the elected movie.

Similarly TripAdvisor provides the information about the hotels, flights and holiday homes based on the interests of the user such as scuba diving, journey into jungle with suggested locations.

All the above recommender systems provide suggestions to the users based on either item based or user profile based recommender systems. The recommender systems in the system can have the benefit of using memory and processor of the system but the current work is dealing with mobile based recommender systems along with the existing methods and research issues and future scope of the work.

The flow of the work is in section II the various mobile recommender systems in the literature were presented. Section III describes the research gaps/issues in the existing mobile based recommender systems. Section IV gives the conclusion and future scope of the work.

\section{VARIOUS MOBILE RECOMMENDER SYSTEMS}

Personalized recommendation systems gaining popularity as the public were using the mobiles rapidly rather than the machines. If multiple users are providing the similar kind of the preferences and the usage of Collaborative Filtering for the accurate recommendations. 
The issue here is if any user intentionally want to promote or damage the identified product and trying to influence the behavior of the user by giving wrong ratings or recommendations. The issue greatly affects the accuracy of the recommendation system and gives negative suggestions to the user.

The research gap in this approach is identifying fake profiles and analyze the wrong postings related to the place or product or person.

The other recommender system which is considering the location based suggestions like based on the user location some items might be famous in that area. The point is as per the studies there is close association between consumer buying habits and location. The best example we can see now a days while using Facebook is if any friend near to your location then the app notifies about the same thing to you.

The other recommendation system is based on the user emotions the recommender system should provide the corresponding product or place so as to refresh as early as possible. The artificial intelligence based emotion recognition helps the users to capture the happy mood or sorrow or anxiety or angry etc., based on the emotions the system should behave with the user.

The recommender system in the food ordering system such as Swiggy and Zomato offers us various recommendations such as the hotels information with ratings and availability. After selection of the hotel provides a menu of items along with rating and must try information of the food products.

Especially for vegetarian members there is an option of the avoiding non vegetarian items and only displays the veg based classification of the food. The recommendation involve rating and must try.

The issue with the recommendation is there is no comparative analysis between the hotels available such as in case of ratings of the food, price and must try kind of the parameters.

The research gap is to establish an option like selection of multiple hotels for the same products and coming up with rating and prices so that the users can have the benefits of the price and taste which in turn improves the quality of the food.

\section{PROPOSED METHOD OF MOBILE BASED RECOMMENDER SYSTEM \& RESULTS}

The above description about the recommender systems has given various insights of the development of the mobile based recommender systems.

The proposed method consider the parameters of the device and accordingly the content gets loaded. The identification of the fake and intentional reviews or promotions can be identified by a spam filtering approach such as Naïve Bayes classification and suggest the users by avoiding these kind of the recommendations.

The other implementation parameter here is in case of location based systems there is only option like up to some minutes or hours only the user can hide the identity to the friend list. By enabling the option of hiding the location sharing of the user one can have the privacy preserving based recommender system.

The third possible research implementation provided in this work is comparative analysis of various products along with ratings and price especially in the food ordering apps.
This can be achieved with the provision of selecting the multiple labels and the corresponding products in the different labels by gathering the ratings and price data along with location based preference system

The problem here is the same label may not doing well in another location for the identified product, based on this convention the system can capture the reviews exclusively form the location of the user so that the final ratings were based on the quality and location based. The outcome is significant as it is two fold of the product review along with the location based preference of the user.

The need of the mobile based recommender system based on the user profile, location based along with ratings gives an accurate form of the suggestions. The existing recommendations gives the global ratings of the products not particular to the location leads to the bias of the product which is not the goal of the recommender system.

The other feature which was not focused in the existing recommender systems is comparative analysis of the different labels with same product line information. The feature enables the better selection mechanism of the products by the user.

The integration of all the above mentioned points can be put together in the form of algorithmic notation which provides a systematic approach for the better recommender system

A simple algorithms is proposed so as to achieve the above conventions

Algorithm Mob_Recom_Loc (User_Profile, Loc, Ratings) Input: User_Profile, Location, Ratings

Output: Recommendations/Suggestions

Start

1. Capture the device profile so as to load the recommender system accordingly.

2. Identify the location

3. Post the product/place/item information

4. Get the ratings based on the analysis of the product related to the location of the user.

5. Eliminate the ratings given by the users other than the current location so as to come up with exact ratings of the product

6. $\quad$ Display the suggestion captured in step 5.

7. Integrate the different labels for the same product and capture the ratings and other reviews recorded according to the location.

8. Generate a report with comparative analysis of rating and price Stop

\section{CONCLUSION}

The current work identified some of the existing works related to mobile based recommender system in the context of the user profile, location based specific. The existing works were described and identified some of the issues and research gaps. The work provided the usage of the location and user profile based recommendation system and described the advantages while working with products from different labels.
Blue Eyes Intelligence Engineering

\& Sciences Publication 
The integration of the user profile along with location based recommendation in the algorithmic orientation has been given and concluded with a recommendation to the user.

\section{REFERENCES}

1. Martín, S.S.; López-Catalán, B.; Ramón-Jerónimo, M.A. Factors determining firms' perceived performance of mobile commerce. Ind. Manag. Data Syst. 2012, 112, 946-963.

2. Guo, Y.; Wang, M.X.; Li, X. Application of an improved Apriori algorithm in a mobile e-commerce recommendation system. Ind. Manag. Data Syst. 2017, 117, 287-303.

3. Ansari, A.; Essegaier, S.; Kohli, R. Internet recommendation systems. J. Market. Res. 2000, 37, 363-375.

4. Bodapati, A.V. Recommendation systems with purchase data. J. Market. Res. 2008, 45, 77-93.

5. Jiang, Y.C.; Shang, J.; Liu, Y.Z. Maximizing customer satisfaction through an online recommendation system: A novel associative classification model. Decis. Support Syst. 2010, 48, 470-479.

6. Zhao, Y.S.; Liu, Y.P.; Zeng, Q.A. A weight-based item recommendation approach for electronic commerce systems. Electron. Commer. Res. 2015, 19, 1-22.

7. Zenebe, A.; Norcio, A.F. Representation, similarity measures and aggregation methods using fuzzy sets for content-based recommender systems. Fuzzy Sets Syst. 2009, 160, 76-94.

8. Lin, Z.J. An empirical investigation of user and system recommendations in e-commerce. Decis. Support Syst. 2014, 68, 111-124.

9. Herlocker, J.L.; Konstan, J.A.; Loren, J.; Terveen, G.; Riedl, T. Collaborative filtering recommender systems. ACM Trans. Inf. Syst. 2004, 22, 5-53.

10. Liu, Y.Z.; Jiang, Y.C.; Liu, X.; Yang, S.L. CSMC: A combination strategy for multi-class classification based on multiple association rules. Knowl.-Based Syst. 2008, 21, 786-793.

11. Cho, Y.H.; Kim, J.K.; Kim, S.H. A personalized recommender system based on web usage mining and decision tree induction. Expert Syst. Appl. 2002, 23, 329-342.

12. Rak, R.; Kurgan, L.; Reformat, M. A tree-projection-based algorithm for multi-label recurrent-item associative-classification rule generation. Data Knowl. Eng. 2008, 64, 171-197. 